\title{
LENGTH OF HOSPITAL STAYS OF PATIENTS OPERATED DUE TO GLIOMA TUMOURS IN NEUROSURGERY CLINICS AND INTENSIVE CARE UNITS
}

\author{
Irmak İrem Özyiğit ${ }^{1}$ (D), Fatih Erkan Akay ${ }^{1}$ (D), Elif Cengiz ${ }^{1}$ (D), Janset Özdemir ${ }^{1}$ (D), Pınar Tuncer $^{1}$ (D), \\ Eylül Şenödeyici ${ }^{1}$ (D), Sarper Kızılkaya ${ }^{1}$ (D), Ahmet Tolgay Akıncı ${ }^{2}$ (D)
}

${ }^{1}$ Trakya University School of Medicine, Edirne, TURKEY

${ }^{2}$ Department of Neurosurgery, Trakya University School of Medicine, Edirne, TURKEY

\begin{abstract}
Aims: To evaluate the relationship between the length of hospital stay and age, gender, and disease characteristics among glioma patients who went under surgery in Trakya University School of Medicine. Methods: The data of 51 glioma patients over 18 years of age, who have been followed up during 2019-2020 in Trakya University School of Medicine, Department of Neurosurgery were analyzed. Patients' data comprised of sex, age, tumour location, grades of the tumours, the presence of isocitrate dehydrogenase mutation, whether the patients were hospitalized in the intensive care unit or the neurosurgery clinic, duration of hospitalization, and whether radiotherapy and chemotherapy was received. Length of hospital stay was evaluated separately as intensive care unit and the neurosurgery clinic. Results: Out of 51 patients diagnosed with glioma, 18 (35.3\%) were female, and 33 (64.7\%) were male. The length of neurosurgery clinic and intensive care unit stays were not associated with radiotherapy and chemotherapy approaches. There was a statistically significant difference between the male and female patients in terms of the number of days stayed in the neurosurgery clinic. Conclusion: In conclusion, gender affected the length of neurosurgery clinic stays with a longer duration for female patients in our clinic. A waste number of parameters, including social ones, affect hospital stays. To reveal predictors of postoperative hospitalization thoroughly and overcome the study's limitations, further prospective studies with larger sample sizes are needed. Keywords: Glioma, hospitalization, isocitrate dehydrogenase, retrospective study
\end{abstract}

\section{INTRODUCTION}

Gliomas are the most frequent form of primary intracranial neoplasms, accounting for $81 \%$ of malignant brain neoplasms (1, 2 ). With an incidence rate of 6 per 100,000 individuals in the United States and a peak between the ages of 45-60 years, gliomas typically appear in adults $(2,3)$. Glioma is a general term that refers to neuroepithelial tumours that are mitotically active with a propensity to infiltrate diffusely, arising from supporting cells of the central nervous system $(2,4)$.

Based on microscopic similarities in their supposed cell origins, gliomas are classified into two subtypes: diffuse gliomas are the most common type characterized by broad infiltrative growth into the surrounding parenchyma and develop mainly in elderly patients (5). These gliomas, among which glioblastoma is the most common and deadliest representative, have been described as diffuse astrocytomas (5). The latter ones are non-diffuse gliomas, which are more circumscribed, considerably rarer, and develop mainly in younger patients $(4,5)$. Pilocytic astrocytoma and ependymomas are the most common varieties of this group $(4,5)$. Gliomas can also be classified based on their cell morphologies, mitotic activities, and molecular markers as low grade, high grade, and atypical (2).
The majority of gliomas are not inherited and occur spontaneously (3). Less than $5 \%$ of gliomas are considered hereditary $(3,4)$. Potential risk factors have been studied, and in most cases, the cause of the tumour is unknown (3). The only well-established risk factor for brain neoplasms is exposure to high or moderate ionising radiation doses $(2,4,6)$. It has been shown that low-grade gliomas can progress into high-grade gliomas by changing their genetic makeup, probably due to their exposure to toxicokinetics (2). Furthermore, several studies suggest that having an atopic disease or allergy can decrease the risk of developing glioma $(4,6)$.

While gliomas can appear in all lobes of the brain, mostly in the frontal lobe (23.6\% of gliomas), they can be seen in the brain stem, cerebellum, and spinal cord as well (4). Clinical manifestations depend on the localisation of the mass; however, the most common symptom is headache followed by seizures, nausea, vomiting, change in vision, tingling sensations, and difficulty in ambulation (2). The patients may be neurologically intact or show different degrees of focal weaknesses, sensory deficits, or altered mental status due to the mass effect of tumour growth in severe cases (2).

Clinical diagnosis is commonly achieved using computed tomography and magnetic resonance imaging scans $(2,7)$. Due to its poor prognosis, gliomas require urgent treatment, challenging both the physician and the patient $(7,8)$. Current standard treatments for 
gliomas involve maximum surgical resection followed by chemotherapy (generally temozolomide) and radiotherapy $(2,7,8)$. The treatment success depends on several factors including the time of diagnosis, new-onset or recurrence of the disease, the performance status, and the age of the patient (9). Treatment choice mainly depends on the patient's age as the elderly have lower survival rates and higher risks of toxicity (10).

Patients who undergo craniotomy for primary brain neoplasms are hospitalized, ranging from 4 to 10 days after surgery, and the median duration of stay for a single acute care visit is five days (1114). The length of hospital stay depends on the postoperative complications and the patients' neurological conditions (13). Furthermore, most elderly patients spend their limited time hospitalized (14). Considering the heavy burden of the disease, the predictors of hospitalization in glioma patients are far from clear, and further research is recommended on factors affecting the length of hospital stay $(4,15)$.

This retrospective study aims to evaluate the relationship between the length of hospital stay and age, gender, and disease characteristics among glioma patients who went under surgery in Trakya University School of Medicine.

\section{MATERIAL AND METHODS}

The Institutional Research Ethics Committee approved this retrospective study (Protocol Code: TÜTF-BAEK 2021/169). The digital medical records of patients were screened. Our study group consisted of 51 glioma patients over 18 years of age, who have been followed up during 2019-2020 in Trakya University School of Medicine Department of Neurosurgery. Since all patients who suit the inclusion criteria in our center were included in the study, no sampling methods were used. Patients' data comprised of sex, age, tumour location, tumour grade according to the 2016 World Health Organization (WHO) Classification of Tumours of the Central Nervous System, presence of isocitrate dehydrogenase (IDH) mutation, whether the patients were hospitalized in the intensive care unit (ICU) or the neurosurgery clinic (NRC), duration of hospitalization, and whether radiotherapy, and chemotherapy were received.

Patients' ages at the date of admission were recorded in years. Length of hospital stay was evaluated separately for both in ICU and NRC. For categorical parameters, patients' sex, IDH status, tumour location, WHO grades of the tumours, radiotherapy and chemotherapy treatments were noted.

The data were analyzed using SPSS 23.0.0.0 program. A p-value of $<0.05$ was set for statistical significance. Numbers, percentages, median, and interquartile range (IQR) were used as the descriptive statistics. Shapiro-Wilk test was used to test the normality of variables. Mann-Whitney U test was used to assess non-parametric variables, which were expressed as "median (IQR)". Fisher-Freeman-Halton exact tests were used in the comparison of tumour locations and WHO grades between males and females.

Median values of length of stay in ICU and NRC were analyzed and compared between patients grouped according to their gender, IDH mutation status, chemotherapy, and radiotherapy parameters. Tumour locations and WHO grades of the tumours were analyzed and compared between female and male patients.

\section{RESULTS}

In this retrospective study, out of 51 patients diagnosed with glioma, 18 (35.3\%) were female and 33 (64.7\%) were male. The median (IQR) age of the patients at admission was 60 (15) years.
The median (IQR) stay at NRC was 12 (10) days, while the median (IQR) stay at ICU was 2 (1) days.

Out of 51 patients, $8(15.7 \%)$ patients received chemotherapy (2 patients received only chemotherapy, 6 patients received both treatments), and $32(62.7 \%)$ patients received radiotherapy (26 patients received only radiotherapy, 6 patients received both treatments). 3 (5.9\%) patients' data regarding chemotherapy were missing. Duration of NRC and ICU stays were shorter in the patients who received radiotherapy compared to patients who did not receive radiotherapy. Age and duration of ICU and NRC hospitalization parameters were not normally distributed.

Patients who received radiotherapy had no statistically significant difference in terms of NRC and ICU stays than the ones who did not receive radiotherapy $(\mathrm{p}=0.184, \mathrm{p}=0.179$, respectively). In a similar trend, patients who received radiotherapy stayed in the $\mathrm{NRC}$ and ICU for fewer days than the patients who did not receive radiotherapy. Furthermore, there was a significant difference between the male and female patients regarding the number of days stayed in NRC $(p=0.006)$. In our study, the IDH mutation marker was also examined and it was found that the majority $(70.6 \%)$ of our patients had a negative IDH status. IDH negative patients stayed in ICU for a median of 12 days while IDH positive patients stayed in ICU for 2 days. The data regarding IDH status of 5 (9.8\%) patients were missing. The summary of patients' statistics for the parameters of gender, radiotherapy, chemotherapy, and IDH are presented in Table 1.

Location-based classification showed that the parietal lobe of the brain was the most common location of glial tumours with a prevalence of $25.5 \%$, in male patients. The patients were also classified according to the WHO classification. A summary of the statistical distribution of location and grade of the tumours with regards to gender is shown in Table 2.

Table 1: Summary of patients' statistics for gender, radiotherapy, chemotherapy, and IDH parameters.

\begin{tabular}{|c|c|c|c|c|}
\hline & $\begin{array}{l}\text { Patients } \\
{[n(\%)]}\end{array}$ & $\begin{array}{c}\text { Age } \\
\text { (years) }\end{array}$ & $\begin{array}{l}N R C^{\star} \\
\text { (days) }\end{array}$ & $\begin{array}{l}I C U^{*} \\
\text { (days) }\end{array}$ \\
\hline \multicolumn{5}{|l|}{ Gender } \\
\hline Female & $18(35.3)$ & $58.5(20.2)$ & $16.5(8.5)$ & $2(2)$ \\
\hline Male & $33(64.7)$ & $60(13)$ & $9(7)$ & $2(0)$ \\
\hline $\mathrm{p}$-value & & 0.567 & 0.006 & 0.171 \\
\hline \multicolumn{5}{|l|}{ Radiotherapy } \\
\hline Not received & $19(37.3)$ & $55(14)$ & $15(11.5)$ & $2(0.5)$ \\
\hline Received & $32(62.7)$ & $64.5(12.5)$ & $10.5(8.3)$ & $2(2)$ \\
\hline $\mathrm{p}$-value & & 0.088 & 0.184 & 0.179 \\
\hline \multicolumn{5}{|l|}{ Chemotherapy } \\
\hline Not received & $40(78.4)$ & $58.5(16.2)$ & $12.5(11.3)$ & $2(2.25)$ \\
\hline Received & $8(15.7)$ & $61.5(12)$ & $8(5.75)$ & $2(0.5)$ \\
\hline $\mathrm{p}$-value & & 0.857 & 0.157 & 0.731 \\
\hline \multicolumn{5}{|l|}{ IDH mutation } \\
\hline Negative & $36(70.6)$ & $64.5(13.5)$ & $12(8)$ & $2(0.5)$ \\
\hline Positive & $10(19.6)$ & $55.5(5.5)$ & $12.5(13.75)$ & $2(3.25)$ \\
\hline $\mathrm{p}$-value & & 0.136 & 0.650 & 0.291 \\
\hline Total & $51(100.0)$ & $60(15)$ & $12(10)$ & $2(1)$ \\
\hline
\end{tabular}

IDH: Isocitrate dehydrogenase, NRC: Neurosurgery clinic,

ICU: Intensive care unit

*Data were expressed as "median (IQR)".

Significant value is marked as bold. 
Table 2: Distribution of location and grade (according to WHO classification of glial tumours of the brain) of the tumors by gender.

\begin{tabular}{lccc} 
& Female $[\boldsymbol{n}(\%)]$ & Male [n (\%)] & P-value \\
\hline Tumour locations & & & 0.851 \\
Frontal & $6(11.8)$ & $7(13.7)$ & \\
Temporal & $5(9.8)$ & $8(15.7)$ & \\
Parietal & $6(11.8)$ & $13(25.5)$ & \\
Occipital & $1(2.0)$ & $4(7.8)$ & \\
Cerebellar & $0(0.0)$ & $1(2.0)$ & \\
WHO grades & & & 0.558 \\
II & $1(2.0)$ & $2(3.9)$ & \\
III & $2(3.9)$ & $1(2.0)$ & \\
IV & $12(23.5)$ & $24(47.1)$ & \\
Missing & $3(5.9)$ & $6(11.8)$ & \\
\hline
\end{tabular}

WHO: World Health Organization

\section{DISCUSSION}

Although gliomas are the most frequent malignant brain neoplasms, there are not many studies on the hospitalization of glioma patients. Therefore, in this study, duration of NRC and ICU stays, whether patients received radiotherapy and chemotherapy, location and WHO grades of tumours and IDH mutation statuses were primarily analyzed.

Gliomas are commonly diagnosed in elderly individuals, similar to the rest of the brain tumors (4). The median (IQR) age in our study was 60 (15) years, which is in line with the results of Rahman et al. (15), which reported a median age of 59 (range: 23-90) years. Similar median age results were found in other studies $(13,17)$. However, studies of Ben Nasr et al. (16) and Jan et al. (18) revealed slightly younger mean values of age, possibly due to the inclusion of patients under 18 years old in their studies. Furthermore, results similar to our study were obtained by Rahman et al. (15), and Jan et al. (18) regarding gender. In our study, $35.3 \%$ of the patients were female, whereas female patient percentages of the mentioned studies were $39.3 \%$ and $41.6 \%$, respectively $(15,18)$. These two studies included higher numbers of patients than our study.

To categorise gliomas, the 2016 WHO classification was used from grade I through grade IV. In our study group, there was no patient with a grade I glioma. There were $24(47.1 \%)$ male and 12 (23.5\%) female patients with a diagnosis of grade IV glioma, each representing the majority in their gender groups. It is known that longer survival durations are associated with grade II and III gliomas, which are accompanied by IDH mutations (4). Since no survival analysis was conducted in our study, we cannot compare it with the current literature. In our research, the number of grade IV tumours was higher, and IDH statuses of patients were mostly negative, which is in line with the aforementioned literature.

In our study, tumours were also categorized based on their locations inside the brain. Six different locations were identified. The most common tumour location was the parietal lobe $(25.5 \%)$ for male patients while frontal (11.8\%) and parietal (11.8\%) lobes were the most common locations for female patients. In contrast, in a study by Verlut et al. (17), the most common area of lesions was the frontal lobe in the overall study population. Another study reported frontal (35.2\%) and temporal (33.2\%) lobes as the most common (15).
Following surgical management of gliomas, radiotherapy and chemotherapy are the main treatment approaches (9). In our study, patients who received radiotherapy outnumbered the patients who received chemotherapy. Also, out of 8 patients with chemotherapy treatment, 6 of them received radiotherapy together with chemotherapy. A phase III trial study by Malmström et al. (10) revealed that temozolomide chemotherapy had better outcomes than standard radiotherapy. However, only elderly patients were included in this study, and this study did not evaluate the length of NRC and ICU stays as an outcome. Contrastly, our study evaluated the length of hospitalization and found that the choice of chemotherapy treatment did not lead to better outcomes in terms of the length of hospitalization. In a study conducted on glioblastoma patients, the number of patients who received radiotherapy alone was higher, which is similar to our results (19).

Duration of NRC and ICU stays were found to be unrelated to the type of treatment in our study. However, there was a difference between female and male patients regarding the lengths of NRC stays. Muhlestein et al. (12) and Dasenbrock et al. (13) also reported a difference between the sexes. Nevertheless, in their studies with much larger samples, female patients stayed a lesser number of days, unlike in our study. We think that many factors affecting hospitalization, including social factors, might have an impact on this result, as well as major limitations of the study, which are small sample size, retrospective construct, and the fact that only stays in NRC and ICU and not other services are accounted for. The median length of stays was 12 days in NRC and 2 days in ICU. A study by Moroney et al. (19) reported a median of 14 days for acute hospitalization after surgery. The majority of the patients were inpatients for a minimum of 14 days in another study, which is higher than our results (14). In another study, the mean of hospitalization was 6.9 days and the duration decreased in patients who received a biopsy (18). However, our study did not specify surgery techniques to compare with this result. In another study, records of inpatients showed less than eight days for $72.2 \%$ of their patients, which is less than our results (13).

This study has some limitations. Our analysis included a limited number of patients $(n=51)$. A more extensive period can be analyzed or multi-centre studies can be conducted in upcoming research studies to meet current articles' results. Further studies including survival analysis, number of emergency visits, and the cost of care per patient are needed to improve our findings.

In conclusion, our study provided general information on hospitalization and its related factors. The length of NRC and ICU stays were not associated with radiotherapy and chemotherapy approaches. However, gender affected the length of NRC stays, with a longer duration for female patients. Locations of tumours varied, while the WHO grades were mainly graded IV in both genders. Further studies are needed to overcome limitations and to reveal predictors of postoperative hospitalization thoroughly.

Ethics Committee Approval: This study was approved by the Scientific Research Ethics Committee of Trakya University School of Medicine (Protocol Code: TÜTF-BAEK 2021/169).

Informed Consent: Informed consent was obtained from participants.

Conflict of Interest: The authors declare no conflict of interest.

Author Contributions: Concept: IİO,, FEA, EC, JÖ, PT, EŞ, SK, ATA. Design: İÖ, FEA, EC, JÖ, PT, EŞ, SK, ATA. Supervision: IIIÖ, FEA, EC, JÖ, PT, EŞ, SK, ATA. Resources: IIIÖ, FEA, EC, JÖ, PT, EŞ, SK, ATA. Materials: IIIÖ, FEA, EC, JÖ, PT, EŞ, SK, ATA. Data collection and/or processing: IİÖ, FEA, EC, JÖ, PT, EŞ, SK, ATA. Analysis and/or Interpretation: IIÖ, FEA, EC, JÖ, PT, EŞ, SK, ATA. Literature Search: IİÖ, FEA, EC, JÖ, PT, EŞ, SK, ATA. Writing Manuscript: IIIÖ, FEA, EC, JÖ, PT, EŞ, SK, ATA. Critical Review: IIÖ, FEA, EC, JÖ, PT, EŞ, SK, ATA. 
Financial Disclosure: The authors declared that this study received no financial support.

Editor-in-Chief's Note: Seven authors of this article, Irmak İrem Özyiğit, Fatih Erkan Akay, Elif Cengiz, Janset Özdemir, Pınar Tuncer, Eylül Șenödeyici and Sarper Kizllkaya are members of the editorial board of Turkish Medical Student Journal. However, they did not take place in any stage of the editorial decision of the manuscript. The editors who evaluated this manuscript are from other institutions.

\section{REFERENCES}

1. Ostrom QT, Bauchet L, Davis FG et al. The epidemiology of glioma in adults: a "state of the science" review. Neuro Oncol 2014;16(7):896-913.

2. Mesfin FB, Al-Dhahir MA. Gliomas. StatPearls. Treasure Island (FL): StatPearls Publishing; 2021.

3. Schneider T, Mawrin C, Scherlach C et al. Gliomas in adults. Dtsch Arztebl Int 2010;107(45):799-807.

4. Davis ME. Epidemiology and overview of gliomas. Semin Oncol Nurs 2018;34(5):420-9.

5. Wesseling P, Capper D. WHO 2016 classification of gliomas. Neuropathol Appl Neurobiol 2018;44(2):139-50.

6. Ostrom QT, Cote DJ, Ascha $\mathrm{M}$ et al. Adult glioma incidence and survival by race or ethnicity in the United States from 2000 to 2014. JAMA Oncol 2018;4(9):125462 .

7. Batash R, Asna N, Schaffer P et al. Glioblastoma multiforme, diagnosis and treatment; recent literature review. Curr Med Chem 2017;24(27):3002-9.

8. Vega RA, Traylor JI, Patel R et al. Combined surgical resection and laser interstitial thermal therapy for glioblastoma: technical note. J Neurol Surg A Cent Eur Neurosurg 2020;81(4):348-54
9. Alifieris C, Trafalis DT. Glioblastoma multiforme: pathogenesis and treatment. Pharmacol Ther 2015;152:63-82.

10. Malmström A, Grønberg BH, Marosi C et al. Temozolomide versus standard 6-week radiotherapy versus hypofractionated radiotherapy in patients older than 60 years with glioblastoma: the Nordic randomised, phase 3 trial. Lancet Oncol 2012;13(9):916-26.

11. Wasilewski A, Serventi J, Kamalyan L et al. Acute care in glioblastoma: the burden and the consequences. Neurooncol Pract 2017;4(4):248-54.

12. Muhlestein WE, Akagi DS, Davies JM et al. Predicting inpatient length of stay after brain tumor surgery: developing machine learning ensembles to improve predictive performance. Neurosurgery 2019;85(3):384-93.

13. Dasenbrock HH, Liu KX, Devine CA et al. Length of hospital stay after craniotomy for tumor: a National Surgical Quality Improvement Program analysis. Neurosurg Focus 2015;39(6):E12.

14. Arvold ND, Wang Y, Zigler C et al. Hospitalisation burden and survival among older glioblastoma patients. Neuro Oncol 2014;16(11):1530-40.

15. Rahman R, Catalano PJ, Reardon DA et al. Incidence, risk factors, and reasons for hospitalization among glioblastoma patients receiving chemoradiation. J Neurooncol 2015;124(1):137-46.

16. Ben Nasr S, Haddaoui A, Bach Hamba S et al. Glioblastoma in Tunisia: a retrospective study about 41 cases. Tunis Med 2015;93(10):598-601.

17. Verlut C, Mouillet G, Magnin E et al. Age, neurological status MRC scale, and postoperative morbidity are prognostic factors in patients with glioblastoma treated by chemoradiotherapy. Clin Med Insights Oncol 2016;10:77-82.

18. Jan BM, Sarmast AH, Bhat AR et al. Profile of high grade gliomas - a single center experience. Gulf J Oncolog 2018;1(26):27-32.

19. Moroney C, Perry JR, Tsang DS et al. Hospitalizations in elderly glioblastoma patients. Ann Palliat Med 2017;6(Suppl 2):161-9. 